\title{
Channel state information estimation for 5G wireless communication systems: Recurrent neural networks approach
}

\author{
Mohamed Hassan Ali ${ }^{\text {Corresp., }}{ }^{1}$, Ibrahim B. M. Taha ${ }^{2}$ \\ 1 Department of Electrical Engineering, Faculty of Engineering, Al-Azhar University,, 1Department of Electrical Engineering, Faculty of Engineering, Al- \\ Azhar University, Qena 83513, Egypt, Qena, Qena, Egypt \\ 2 Department of Electrical Engineering, College of Engineering,, Taif University, Taif 21944,, Saudi Arabia, Taif, Taif \\ Corresponding Author: Mohamed Hassan Ali \\ Email address: mhessai@azhar.edu.eg
}

In this study, a deep learning bidirectional long short-term memory (BiLSTM) recurrent neural network-based channel state information estimator is proposed for $5 \mathrm{G}$ orthogonal frequency-division multiplexing systems. The proposed estimator is a pilot-dependent estimator and follows the online learning approach in the training phase and the offline approach in the practical implementation phase. The estimator does not deal with complete a priory certainty for channels' statistics and attains superior performance in the presence of a limited number of pilots. A comparative study is conducted using three loss functions, namely, mean absolute error, cross entropy function for kth mutually exclusive classes and sum of squared of the errors. The Adam optimisation algorithm is used to evaluate the performance of the proposed estimator under each loss function. In terms of symbol error rate and accuracy metrics, the proposed estimator outperforms long shortterm memory (LSTM) neural network-based channel state information, least squares and minimum mean square error estimators under different simulation conditions. The computational and training time complexities for deep learning BiLSTM- and LSTM-based estimators are provided. Given that the proposed estimator relies on the deep learning neural network approach, where it can analyse massive data, recognise statistical dependencies and characteristics, develop relationships between features and generalise the accrued knowledge for new datasets that it has not seen before, the approach is promising for any $5 \mathrm{G}$ and beyond communication system. 
1 Channel State Information Estimation for 5G Wireless

2 Communication Systems: Recurrent Neural Networks

3 Approach

${ }^{1}$ Department of Electrical Engineering, Faculty of Engineering, Al-Azhar University, Qena 83513, Egypt

${ }^{2}$ Department of Electrical Engineering, College of Engineering, Taif University, Taif 21944, Saudi Arabia

Corresponding Author:

Mohamed Hassan Essai Ali ${ }^{1}$

Masaken Osman, Qena, Qena, 83513, Egypt

Email address: mhessai@azhar.edu.eg

\section{Abstract}

In this study, a deep learning bidirectional long short-term memory (BiLSTM) recurrent neural network-based channel state information estimator is proposed for $5 \mathrm{G}$ orthogonal frequencydivision multiplexing systems. The proposed estimator is a pilot-dependent estimator and follows the online learning approach in the training phase and the offline approach in the practical implementation phase. The estimator does not deal with complete a priory certainty for channels' statistics and attains superior performance in the presence of a limited number of pilots. A comparative study is conducted using three classification layers that use loss functions: mean absolute error, cross entropy function for kth mutually exclusive classes and sum of squared of the errors. The Adam, RMSProp, SGdm, and Adadelat optimisation algorithms are used to evaluate the performance of the proposed estimator using each classification layer. In terms of symbol error rate and accuracy metrics, the proposed estimator outperforms long short-term memory (LSTM) neural network-based channel state information, least squares and minimum mean square error estimators under different simulation conditions. The computational and training time complexities for deep learning BiLSTM- and LSTM-based estimators are provided. Given that the proposed estimator relies on the deep learning neural network approach, where it can analyse massive data, recognise statistical dependencies and characteristics, develop relationships between features and generalise the accrued knowledge for new datasets that it has not seen before, the approach is promising for any $5 \mathrm{G}$ and beyond communication system. 
40

41

42

43

44

45

46

47

48

49

50

51

52

53

54

55

56

57

58

59

60

61

62

63

64

65

66

67

68

69

70

71

72

73

74

75

76

77

78

79

80

81

82

83

84

85

86

87

88

89

90

91

\section{Introduction}

$5 \mathrm{G}$ wireless communication is the most active area of technology development and a rapidly growing branch of the wider field of communication systems. Wireless communication has made various possible services ranging from voice to multimedia.

The physical characteristics of the wireless communication channel and many unknown surrounding effects result in imperfections in the transmitted signals. For example, the transmitted signals experience reflections, diffractions, and scattering, which produce multipath signals with different delays, phase shift, attenuation, and distortion arriving at the receiving end; hence, they adversely affect the recovered signals (Oyerinde \& Mneney 2012b).

A priori information on the physical characteristics of the channel provided by pilots is one of the significant factors that determine the efficiency of channel state information estimators (CSIEs). For instance, if not a priori information is available (no or insufficient pilots), channel estimation is useless; finding what you do not know is impossible. When complete information on the transmission channel is available, CSIEs are no longer needed. Thus, a priori uncertainty exists for communication channel statistics. However, the classical theory of detection, recognition, and estimation of signals deals with complete priory certainty for channel statistics, and it is an unreliable and unpractical assumption (Bogdanovich et al. 2009).

In the classic case, uncertainty is related to useful signals. In detection problems, the unknown is the fact of a signal existence. In recognition problems, the unknown is the type of signal being received at the current moment. In estimation problems, the unknown is the amplitude of the measured signal or one of its parameters. The rest of the components of the signal-noise environment in classical theory are regarded as a priori certain (known) as follows: the known is the statistical description of the noise, the known is the values of the unmeasured parameters of the signal and the known is the physical characteristics of the wireless communication channel. In such conditions, the classical theory allows the synthesis of optimal estimation algorithms, but the structure and quality coefficients of the algorithms depend on the values of the parameters of the signal-noise environment. If the values of the parameters describing the signal-noise environment are slightly different from the parameters for which the optimal algorithm is built, then the quality coefficients will become substantially poor, making the algorithm useless in several cases (Bogdanovich et al. 2009), (O'Shea et al. 2017). The most frequently used CSIEs are derived from signal and channel statistical models by employing techniques, such as maximum likelihood (ML), least squares (LS), and minimum mean squared error (MMSE) optimisation metrics (Kim 2015).

One of the major concerns in the optimum performance of wireless communication systems is providing accurate channel state information (CSI) at the receiver end of the systems to detect the transmitted signal coherently. If CSI is unavailable at the receiver end, then the transmitted signal can only be demodulated and detected by a noncoherent technique, such as differential demodulation. However, using a noncoherent detection method occurs at the expense of a loss of signal-to-noise ratio of about 3-4 dB compared with using a coherent detection technique. To eliminate such losses, researchers have focused on the development of channel estimation techniques to provide perfect detection of transmitted information in wireless communication systems using the Orthogonal Frequency-Division Multiplexing (OFDM) modulation scheme (Oyerinde \& Mneney 2012a).

The use of deep learning neural networks (DLNNs) is the state-of-the-art approach in the field of wireless communication. The amazing learning capabilities of DLNNs from training data sets and the tremendous progress of graphical processing units (GPUs), which are considered the most powerful tools for training DLNNs, have motivated its usage for different wireless communication issues, such as modulation recognition (Zhou et al. 2020), (Karra et al. 2017) and channel state estimation and detection (Essai Ali ; Joo et al. 2019; Kang et al. 2020; Ma et al. 2018; Ponnaluru \& Penke 2020; Yang et al. 2019a; Ye et al. 2018). According to (Karra et al. 2017; Kim 2015; Oyerinde \& Mneney 2012a; Zhou et al. 2020) and (Ma et al. 2018), all proposed deep learningbased CSIEs have better performance compared with the examined traditional channel ones, such as LS and MMSE estimators. 
92

93

94

95

96

97

98

99

100

101

102

103

104

105

106

107

108

109

110

111

112

113

114

115

116

117

118

119

120

121

122

123

124

125

126

127

128

129

130

131

132

133

134

135

136

137

138

139

140

141

142

143

144

Recently, numerous long short-term memory (LSTM)- and BiLSTM-based applications have been introduced for prognostic and health management (Zhao et al. 2020), artificial intelligencebased translation systems (Wu et al. 2016), (Ong 2017) and other areas. For channel state information estimation in 5G-OFDM wireless communication systems, many deep learning approaches, such as convolutional neural network $(\mathrm{CNN})$, recurrent neural network (RNN) (e.g. LSTM and BiLSTM NNs) and hybrid (CNN and RNN) neural networks have been used (Essai Ali ; Liao et al. 2019; Luo et al. 2018b; Ponnaluru \& Penke 2020; Yang et al. 2019a; Yang et al. 2019b; Ye et al. 2018).

In (Liao et al. 2019), a deep learning-based CSIE was proposed by using CNN and BiLSTM$\mathrm{NN}$ for the extraction of the feature vectors of the channel response and channel estimation, respectively. The aim was to improve the channel state information estimation performance at the downlink, which is caused by the fast time-varying and varying channel statistical characteristics in high-speed mobility scenarios. In (Luo et al. 2018a), an online-trained CSIE that is an integration of CNN and LSTM-NN was proposed. The authors also developed an offline-online training technique that applies to 5G wireless communication systems. In (Ye et al. 2018), a joint channel estimator and detector that is based on feedforward DLNNs for frequency selective channel (OFDM) systems was introduced. The proposed algorithm was found to be superior to the traditional MMSE estimation method when unknown surrounding effects of communication systems are considered. In (Yang et al. 2019a), an online estimator was developed by adopting feedforward DLNNs for doubly selective channels. The proposed estimator was considered superior to the traditional LMMSE estimator in all investigated scenarios. In (Ponnaluru \& Penke 2020), a one-dimensional CNN (1D-CNN) deep learning estimator was proposed. Under various modulation scenarios and in terms of MSE and BER metrics, the authors compared the performance of the proposed estimator with that of feedforward neural networks (FFNN), MMSE and LS estimators. 1D-CNN outperformed LS, MMSE and FFNN estimators. In (Essai Ali), an online pilot-assisted estimator model for OFDM wireless communication systems was developed by using LSTM NN. The conducted comparative study showed the superior performance of the proposed estimator in comparison with LS and MMSE estimators under limited pilots and a prior uncertainty of channel statistics. The authors in (Sarwar et al. 2020) used the genetic algorithmoptimised artificial neural network to build a CSIE. The proposed estimator was dedicated for space-time block-coding MIMO-OFDM communication systems. The proposed estimator outperformed LS and MMSE estimators in terms of BER at high SNRs, but it achieved approximately the same performance as LS and MMSE estimators at low SNRs. The authors in (Senol et al. 2021) proposed a CSIE for OFDM systems by using ANN under the condition of sparse multipath channels. The proposed estimator achieved a comparable SER performance as matching pursuit- and orthogonal matching pursuit-based estimators at a lower computational complexity than that of the examined estimators. The authors in (Le Ha et al. 2021) proposed a CSIE that uses deep learning and LS estimator and utilizes the multiple-input multiple-output system for 5G-OFDM. The proposed estimator minimizes the MSE loss function between the LSbased channel estimation and the actual channel. The proposed estimator outperformed LS and LMMSE estimators in terms of BER and MSE metrics.

In this study, a BiLSTM DLNN-based CSIE for OFDM wireless communication systems is proposed and implemented. To the best of the authors' knowledge, this work is the first to use the BiLSTM network as a CSIE without integration with CNN. The proposed estimator does not need any prior knowledge of the communication channel statistics and powerfully works at limited pilots (under the condition of less CSI). The proposed BiLSTM-based CSIE is a data-driven estimator, so it can analyse, recognise and understand the statistical characteristics of wireless channels suffering from many known interferences such as adjacent channel, inter symbol, inter user, inter cell, co-channel and electromagnetic interferences and unknown ones (Jeya et al. 2019; Sheikh 2004). Although an impressively wide range of configurations can be found for almost every aspect of deep neural networks, the choice of loss function is underrepresented when addressing communication problems, and most studies and applications simply use the 'log' loss function (Janocha \& Czarnecki 2017). In this study two customed loss functions known as mean

Peer) Comput. Sci. reviewing PDF | (CS-2021:04:60719:2:1:NEW 17 Jul 2021) 
145

146

147

148

149

150

151

152

153

154

155

156

157

158

159

160

161

162

163

164

165

166

167

168

169

170

171

172

173

174

175

176

177

178

179

180

181

182

183

184

185

186

187

188

189

190

191

absolute error (MAE), and sum of squared errors (SSE) are proposed to obtain the most reliable and robust estimator under unknown channel statistical characteristics and limited pilot numbers.

The performance of the proposed BiLSTM-based estimator is compared with the performance of the most frequently used LS and MMSE channel state estimators. The obtained results show that the BiLSTM-based estimator attains a comparable performance as the MMSE estimator and outperforms LS and MMSE estimators at large and small numbers of pilots, respectively. In addition, the proposed estimator improves the transmission data rate of OFDM wireless communication systems because it exhibits optimal performance compared with the examined estimators at a small number of pilots.

The rest of this paper is organised as follows. The DLNN-based CSIE is presented in Section II. The standard OFDM system and the proposed deep learning BiLSTM NN-based CSIE are presented in Section III. The simulation results are given in Section IV. The conclusions and future work directions are provided in Section V.

\section{DLNN-BASED CSIE}

In this section, a deep learning BiLSTM NN for channel state information estimation is presented. The BiLSTM network is another version of LSTM neural networks, which are recurrent neural networks $(\mathrm{RNN})$ that can learn the long-term dependencies between the time steps of input data (Hochreiter \& Schmidhuber 1997) (Luo et al. 2018b; Zhao et al. 2020).

The BiLSTM architecture mainly consists of two separate LSTM-NNs and has two propagation directions (forward and backward). The LSTM NN structure consists of input, output and forget gates and a memory cell. The forget and input gates enable the LSTM NN to effectively store longterm memory. Figure 1 shows the main construction of the LSTM cell (Hochreiter \& Schmidhuber 1997). The forget gate enables LSTM NN to remove the undesired information by currently used input ${ }^{x_{t}}$ and cell output $h_{t}$ of the last process. The input gate finds the information that will be used with the previous LSTM cell state $c_{t-1}$ to obtain a new cell state $c_{t}$ based on the current cell input $x_{t}$ and the previous cell output $h_{t-1}$. Using the forget and input gates, LSTM can decide which information is abandoned and which is retained.

The output gate finds current cell output $h_{t}$ by using the previous cell output $h_{t-1}$ at current cell state $c_{t}$ and input $x_{t}$. The mathematical model of the LSTMNN structure can be described through Equations (1) - (6).

$$
\begin{aligned}
& i_{t}=\sigma_{g}\left(w_{i} x_{t}+R_{i} h_{t-1}+b_{i}\right), \\
& f_{t}=\sigma_{g}\left(w_{f} x_{t}+R_{f} h_{t-1}+b_{f}\right), \\
& g_{t}=\sigma_{c}\left(w_{g} x_{t}+R_{g} h_{t-1}+b_{g}\right), \\
& o_{t}=\sigma_{g}\left(w_{o} x_{t}+R_{o} h_{t-1}+b_{o}\right), \\
& c_{t}=f_{t} \odot c_{t-1}+i_{t} \odot g_{t}, \\
& h_{t}=o_{t} \odot \sigma_{c}\left(c_{t}\right),
\end{aligned}
$$

where $i, f, g, o, \sigma_{c}, \sigma_{g}$ and $\odot$ denote the input gate, forget gate, cell candidate, output gate, state activation function (hyperbolic tangent function ( $\tanh$ ), gate activation function (sigmoid function) and Hadamard product (element-wise multiplication of vectors), respectively. $\mathbf{W}=\left[w_{i} w_{f} w_{g} w_{o}\right]^{T}$, $\mathbf{R}=\left[\begin{array}{llll}R_{i} & R_{f} & R_{g} & R_{o}\end{array}\right]^{T}$ and $\mathbf{b}=\left[\begin{array}{llll}b_{i} & b_{f} & b_{g} & b_{o}\end{array}\right]^{T}$ are input weights, recurrent weights and bias, respectively.

LSTM DNN, only analyses the impact of the previous sequence in the present, disregarding information later on and failing to reach optimal performance. On the other hand BiLSTM connects the LSTM unit's output bidirectionally (forward and backward propagation directions) and capture bidirectional signals dependencies, increasing the overall model's performance.

The forward and backward propagation directions of BiLSTM are transmitted at the same time to the output unit. Therefore, old and future information can be captured, as shown in Figure 2. At 
192

193

194

195

196

197

198

199

200

201

202

203

204

205

206

207

208

209

210

211

212

213

214

215

216

217

218

219

220

221

222

223

224

225

226

227

228

229

230

231

232

233

234

235

236

237

238

any time $t$, the input is fed to forward LSTM and backward LSTM networks. The final output of BiLSTM-NN can be expressed as follows:

$$
h_{t}=\overrightarrow{h_{t}} \odot \overleftarrow{h_{t}}
$$

where $\vec{h}_{t}$ and $\overleftarrow{h}_{t}$ are forward and backward outputs of BiLSTM-NN, respectively. The operation of BiLSTM in the proposed estimator can be described briefly by the following algorithm:

Input: sequence represents transmitted signal (original signal + channel model)

Output: Prediction matrix of the extracted features of the input sequence

Step 1: The forward LSTM layer receives the transmitted signal vectors from $X$.

for $i$ length $(X)$ do

send $X_{i}$ to BiLSTM Layer

end for

Step 2: Equations 1-6 are used to update the state of the LSTM cell.

Step 3: The backward LSTM layer receives the signal vectors from $X$, and the two previous steps are repeated.

Step 4: A hidden state sequence vector is created by splicing the forward and backward sequences of hidden layers.

Step 5: A hidden state sequence vector is sent into a full connection layer and the prediction matrix is obtained

Step 6: Return the prediction matrix.

To build the DL BiLSTM NN-based CSIE, an array is created with the following five layers: sequence input, BiLSTM, fully connected, softmax and output classification. The input size was set to 256. The BiLSTM layer consists of 30 hidden units and shows the sequence's last element. Four classes are specified by considering the size 4 fully connected (FC) layer, followed by a softmax layer and ended by a classification layer. Figure 3 illustrates the structure of the proposed estimator (Essai Ali ; Ye et al. 2018).

As the proposed BiLSTM-based CSIE is built, the weights and biases of the proposed estimator are optimised (tuned) using the desired optimisation algorithm. The optimisation algorithm trains the proposed estimator by using one of three loss functions, namely, cross entropy function for $\mathrm{k}^{\text {th }}$ mutually exclusive classes (crossentropyex), mean absolute error (MAE), and sum of squared errors (SSE). The loss function estimates the loss between the expected and actual outcome. During the learning process, optimisation algorithms try to minimise the available loss function to the desired error goal by optimising the DLNN weights and biases iteratively at each training epoch. Figure 4 illustrates the training processes of the proposed estimator. Selecting a loss function is one of the essential and challenging tasks in deep learning. Also, investigating the efficiency of the training process using different optimization algorithms such as Adaptive Moment Estimation (Adam), Root Mean Square Propagation (RMSProp), Stochastic Gradient Descent with momentum (SGdm) (Dogo et al. 2018), and an adaptive learning rate method (Adadelta) (Zeiler 2012). The proposed estimator is trained using above-mentioned three different loss functions and optimization algorithms to obtain the most optimal BiLSTM-based estimator for wireless communication systems with low prior information (limited pilots) for signal-noise environments. 
239

240

241

242

243

244

245

246

247

248

249

250

251

252

253

254

255

256

257

258

259

260

261

262

263

264

265

266

267

268

269

270

271

272

273

274

275

276

277

278

279

280

281

282

283

284

285

286

287

288

The standard OFDM wireless communication system and an offline DL of the proposed CSIE are presented in the following subsections.

\section{OFDM SYSTEM MODEL}

In accordance with (Essai Ali ; Ye et al. 2018), Figure 5 clearly illustrates the structure of the traditional OFDM communication system. On the transmitter side, a serial-to-parallel (S/P) converter is used to convert the transmitted symbols with pilot signals into parallel data streams. Then, inverse discrete Fourier transform (IDFT) is applied to convert the signal into the time domain. A cyclic prefix (CP) must be added to alleviate the effects of inter-symbol interference. The length of the CP must be longer than the maximum spreading delay of the channel.

The multipath channel of a sample space defined by complex random variables $\{h(n)\}_{n=0}^{N-1}$ is considered. Then, the received signal can be evaluated as follows:

$$
y(n)=x(n) \oplus h(n)+w(n),
$$

where $x(n)$ is the input signal, $\oplus$ is circular convolution, $w(n)$ is additive white Gaussian noise $(\mathrm{AWGN})$ and $y(n)$ is the output signal.

The received signal in the frequency domain can be defined as

$$
Y(k)=X(k) H(k)+W(k),
$$

where the discrete Fourier transformations (DFT) of $x(n), h(n), y(n)$ and $w(n)$ are $X(k), H(k)$, $Y(k)$ and $W(k)$, respectively. These discrete Fourier transformations are estimated after removing $\mathrm{CP}$.

The OFDM frame includes the pilot symbols of the $1^{\text {st }}$ OFDM block and the transmitted data of the next OFDM blocks. The channel can be considered stationary during a certain frame, but it can change between different frames. The proposed DL BiLSTM NN-based CSIE receives the arrived data at its input terminal and extracts the transmitted data at its output terminal (Essai Ali), (Ye et al. 2018).

\section{OFFLINE DL OF THE DL BILSTM NN-BASED CSIE}

DLNN utilisation is the state-of-the-art approach in the field of wireless communication, but DLNNs have high computational complexity and long training time. GPUs are the most powerful tools used for training DLNNs (Sharma et al. 2016). Training should be done offline due to the long training time of the proposed CSIE and the large number of BILSTM-NN's parameters, such as biases and weights, that should be tuned during training. The trained CSIE is then used in online implementation to extract the transmitted data (Ye et al. 2018), (Essai Ali).

In offline training, the learning dataset is randomly generated for one subcarrier. The transmitting end sends OFDM frames to the receiving end through the adopted (simulated) channel, where each frame consists of single OFDM pilot symbol and a single OFDM data symbol. The received OFDM signal is extracted based on OFDM frames that are subjected to different channel imperfections.

All classical estimators rely highly on tractable mathematical channel models, which are assumed to be linear, stationary and follow Gaussian statistics. However, practical wireless communication systems have other imperfections and unknown surrounding effects that cannot be tackled well by accurate channel models; therefore, researchers have developed various channel models that effectively characterise practical channel statistics. By using these channel models, reliable and practical training datasets can be obtained by modelling (Bogdanovich et al. 2009), (Essai Ali), (2019).

In this study, the 3GPP TR38.901-5G channel model developed by (2019) is used to simulate the behaviour of a practical wireless channel that can degrade the performance of CSIEs and hence, the overall communication system's performance.

The proposed estimator is trained via the algorithm, which updates the weights and biases by minimising a specific loss function. Simply, a loss function is defined as the difference between 
289

290

291

292

293

294

295

296

297

298

299

300

301

302

303

304

305

306

307

308

309

310

311

312

313

314

315

316

317

318

319

320

321

322

323

324

325

326

327

328

329

330

331

332

333

334

335

the estimator's responses and the original transmitted data. The loss function can be represented by several functions. MATLAB/neural network toolbox allows the user to choose a loss function amongst its available list that contains crossentropyex, MSE, sigmoid and softmax. In this study, another two custom loss functions (MAE and SSE) are created. The performance of the proposed estimator when using three loss functions (i.e. MAE, crossentropyex and SSE) is investigated. The loss functions can be expressed as follows:

$$
\begin{gathered}
\text { crossentropyex }=-\sum_{i=1}^{N} \sum_{j=1}^{c} X_{i j}(k) \log \left(\hat{X}_{i j}(k)\right), \\
\operatorname{MAE}=\frac{\sum_{i=1}^{N} \sum_{j=1}^{c}\left|X_{i j}(k)-\hat{X}_{i j}(k)\right|}{N}, \\
\operatorname{SSE}=\sum_{i=1}^{N} \sum_{j=1}^{c}\left(X_{i j}(k)-\hat{X}_{i j}(k)\right)^{2},
\end{gathered}
$$

where $N$ is the sample number, $C$ is the class number, $X_{i j}$ is the $i^{\text {th }}$ transmitted data sample for the $j^{\text {th }}$ class and $\hat{X}_{i j}$ is the DL BiLSTM-based CSIE response for sample $i$ for class $j$.

Figure 4 illustrates the offline training processes to obtain a learned CSIE based on BiLSTM$\mathrm{NN}$.

\section{Simulation Results}

\section{STUDYING THE PERFORMANCE OF THE PROPOSED, LS AND MMSE ESTIMATORS BY USING DIFFERENT PILOTS AND LOSS FUNCTIONS}

Several simulation experiments are performed to evaluate the performance of the proposed estimator. In terms of symbol error rate (SER) performance analysis, the SER performance of the proposed estimator under various SNRs is compared with that of the LSTM NN-based CSIE (Essai Ali), the well-known LS estimator and the MMSE estimator, which is an optimal estimator but requires channel statistical information. A priori uncertainty of the used channel model statistics is assumed and considered for all conducted experiments.

Moreover, the Adam optimisation algorithm is used to train the proposed estimator whilst using different loss functions to obtain the most robust version of the proposed CSIE. The proposed model is implemented in $2019 \mathrm{~b}$ MATLAB/software.

Table 1 lists the parameters of BiLSTM-NN and LSTM-NN architectures and their related training options. These parameters are identified by a trial-and-error approach. Table 2 lists the parameters of the OFDM system model and the channel model.

The examined estimators' performance is evaluated at different pilot numbers of 4, 8 and 64 as well as crossentropyex, MAE and SSE loss functions. The Adam optimisation algorithm is used for all simulation experiments.

With a sufficiently large number of pilots (64) and the use of the crossentropyex loss function, the proposed BiLSTM crossentropyex $_{\text {estimator outperforms LSTM }}$ crossentropyex, LS and MMSE estimators over the entire SNR range, as shown in Figure 6. At the use of the MAE loss function, the BiLSTM $_{\text {MAE }}$ estimator outperforms the LS estimator over the SNR range [0-18 dB], but LSTM ${ }_{\text {MAE }}$ outperforms it over the SNR range [0-14 dB]. In addition, the BiLSTM ${ }_{M A E}$ and LSTM $_{M A E}$ estimators are at par with the MMSE estimator over the SNR ranges $[0-10 \mathrm{~dB}]$ and $[0-4 \mathrm{~dB}]$, respectively. Beyond these SNR ranges, the MMSE estimator outperforms BiLSTM MAE $_{\text {and }}$ LSTM $_{\mathrm{MAE}}$ estimators. BiLSTM ${ }_{\mathrm{MAE}}$ outperforms LSTM $_{\mathrm{MAE}}$ starting from $0 \mathrm{~dB}$ to $20 \mathrm{~dB}$.

At the use of the SSE loss function, Figure 6 shows that the BiLSTM ${ }_{\text {SSE }}$ and LSTM SSE $_{\text {estimators }}$ achieve approximately the same performance as the MMSE estimator over a low SNR range [0-6 $\mathrm{dB}$. MMSE outperforms the BiLSTM $\mathrm{SSE}_{\text {and }}$ LSTM $_{\mathrm{SSE}}$ estimators starting from $8 \mathrm{~dB}$, and the LS estimator outperforms BiLSTM SSE $_{\text {starting from } 16 \mathrm{~dB} \text { and LSTM }}$ SSE starting from $14 \mathrm{~dB}$. BiLSTM $_{\text {SSE }}$ outperforms LSTM SSE starting from $10 \mathrm{~dB}$ to $20 \mathrm{~dB}$. LS provides poor performance compared with MMSE because it does not use prior information about channel statistics in the 
336

337

338

339

340

341

342

343

344

345

346

347

348

349

350

351

352

353

354

355

356

357

358

359

360

361

362

363

364

365

366

367

368

369

370

371

372

373

374

375

376

377

378

379

380

381

382

383

384

385

386

387

estimation process. MMSE exhibits superior performance, especially with sufficient pilot numbers, because it uses second-order channel statistics. Concisely, MMSE and the proposed BiLSTM $_{\text {crossentropyex }}$ attain close SER performance with respect to all SNRs. Furthermore, at low SNR $(0-6 \mathrm{~dB})$, BiLSTM $_{\text {(crossentropyex, MAE, and SSE)}}, \operatorname{LSTM}_{\text {(crossentropyex, MAE, and SSE) }}$ and MMSE attain approximately the same performance.

Figures 7 present the performance comparison of LS, MMSE, BiLSTM and LSTM-based estimators using the Adam optimisation algorithm and the different (crossentropyex, MAE and SSE) loss functions at 8 pilots. Figure 7 shows that the proposed BiLSTM (crossentropyex, or MAE or SSE) estimators outperform the LSTM (crossentropyex, or MAE or SSE) estimators and the traditional estimators over the examined SNR range. At a low SNR (0-7 dB), the proposed BiLSTM (crossentropyex, or MAE or SSE) estimators exhibit semi-identical performance. Furthermore, the proposed BiLSTM $\mathrm{SSE}$ estimator trained by minimising the SSE loss function outperforms the BiLSTM crossentropyex $_{\text {estimator trained }}$ by minimising the crossentropyex loss function starting from $0 \mathrm{~dB}$; also it outperforms BiLSTM MAE$_{\text {, }}$ which is trained by minimising the MAE loss function starting from $14 \mathrm{~dB}$. Concisely at 8 pilots BiLSTM $_{\text {SSE }}$ estimator achieved the most minimum SER.

Figures 8 show the performance comparison of the LS, MMSE, BiLSTM (crossentropyex, or MAE of SSE) and LSTM $_{\text {(crossentropyex, or MAE or SSE) }}$ estimators at 4 pilots. Figure 8 shows the superiority of the proposed BiLSTM (crossentropyex, or MAE or SSE) estimators in comparison with the traditional estimators, which have lost their workability starting from $0 \mathrm{~dB}$. It also shows the superiority of the proposed estimator BiLSTM $_{\text {(MAE or SSE) }}$ over LSTM $_{\text {(MAE or SSE) }}$. LSTM $_{\text {(crossentropyex) }}$ exhibits a competitive

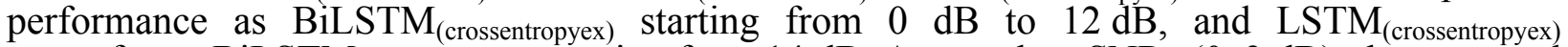
outperforms BiLSTM (crossentropyex) starting from $14 \mathrm{~dB}$. At very low SNRs $(0-3 \mathrm{~dB})$, the proposed BiLSTM $_{\text {(crossentropyex, or MAE or SSE) }}$ estimators have the same performance. The proposed BiLSTM $\mathrm{SSE}$

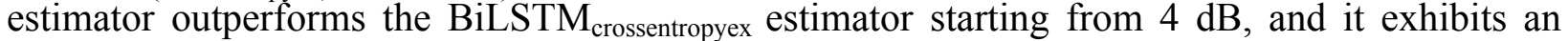
identical performance as the BiLSTM $\mathrm{MAE}$ estimator until $14 \mathrm{~dB}$ and outperforms it in the rest of the SNR examination range.

Figures 6, 7 and 8 emphasise the robustness of the BiLSTM-based estimators against the limited number of pilots, low SNR, and under the condition of a priori uncertainty of channel statistics. They demonstrate the importance of testing various loss functions in the deep learning process to obtain the most optimal architecture of any proposed estimator.

Figure 9 indicates that the proposed BiLSTM crossentropyex BiLSTM $\mathrm{SSE}$ and BiLSTM SSE $_{\text {E }}$ estimators have close SER performance at 64, 8 and 4 pilots, respectively. The performance of BiLSTM $\mathrm{SSE}$ at 8 pilots coincides with the performance of BiLSTM crossentropyex $_{\text {at }} 64$ pilots. Therefore, using the proposed estimators with few pilots is recommended for 5G OFDM wireless communication systems to attain a significant improvement in their transmission data rate. Given that the proposed estimator adopts a training data set-driven approach, it is robust to a priori uncertainty for channel statistics.

\section{LOSS CURVES}

The quality of the DLNNs' training process can be monitored efficiently by exploring the training loss curves. These loss curves provide information on how the training process goes, and the user can decide whether to let the training process continue or stop.

Figures 10-12 show the loss curves of the DLNN-based estimators (BiLSTM and LSTM) at pilot numbers $=64,8$ and 4 and with the three examined loss functions (crossentropyex, MAE and SSE). The curves emphasise and verify the obtained results in Figure 6, 7, and 8. For example, the sub-curves in Figure 10 for BiLSTM crossentropyex $_{\text {and }}$ LSTM $_{\text {crossentropyex }}$ estimators emphasise their superiority over the other estimators. This superiority can be seen clearly from Figures 6. Moreover, the training loss curves in Figures 11 and 12 emphasise the obtained SER performance in Figures 7 and 8, respectively, of each examined DLNN-based CSIE. For more details, good zooming, and analysis of the presented loss curves, they can be downloaded from this link (shorturl.at/lqxGQ). 
388

389

390

391

392

393

394

395

396

397

398

399

400

401

402

403

404

405

406

407

408

409

410

411

412

413

414

415

416

417

418

419

420

421

422

423

424

425

426

427

428

429

430

431

432

433

434

435

436

437

438

439

\section{ACCURACY CALCULATION}

The accuracy of the proposed and other examined estimators is a measure of how the estimators recover transmitted data correctly. Accuracy can be defined as the number of correctly received symbols divided by the total number of transmitted symbols. The proposed estimator is trained in different conditions as indicated in the previous subsection, and we wish to investigate how well it performs in a new data set. Tables 3, 4 and 5 present the obtained accuracies for all examined estimators under all simulation conditions.

As illustrated in Tables 3 to 5, the proposed BiLSTM-based estimator attains accuracies from 98.61 to 100 under different pilots and loss functions. The other examined DL LSTM-based estimator has accuracies from 97.88 to 99.99 under the same examination conditions. The achieved accuracies indicate that the proposed estimator has robustly learned and emphasises the obtained SER performance in Figure 9. The obtained results of MMSE and LS in Tables 1, 2 and 3 emphasise the presented SER performance in Figures 6, 7 and 8, respectively, and show that as the pilot number decreases, the accuracy of the conventional estimators dramatically decreases.

The proposed BiLSTM- and LSTM-based estimators rely on DLNN approaches, where they can analyse huge data sets that may be collected from any plant, recognise the statistical dependencies and characteristics, devise the relationships between features and generalise the accrued knowledge for new data sets that they have not seen before. Thus, they are applicable to any $5 \mathrm{G}$ and beyond communication system.

\section{IMPACT OF USING DIFFERENT OPTIMIZATION ALGORITHMS ON THE PROPOSED ESTMATOR PERFORMANCE}

DL procedures benefit greatly from optimization methods. DNN training can be thought of as an optimisation issue that aims to discover a global optimum by applying gradient descent methods to obtain a robust training, and hence reliable prediction or classification models. Choosing the best optimization method for a particular scientific topic is a difficult task. Using the wrong optimization strategy during training can cause the DN to stay at the local minimum, which results in no training progress (Dogo et al. 2018). As a result, examination is required to evaluate the performance of various optimisers to get the optimal CSIE.

This section provides performance comparison experiments using RMSProp, SGdm, and Adadelta optimisation algorithms (Soydaner \& Intelligence 2020)for training the proposed BiLSTM-based CSIE at using 8-pilots, as illustrated in Fig.13. Table 6 arranges the proposed BiLSTM CSIE estimators using different optimisation algorithms and loss functions from the highest performance to the lowest and their related accuracies.

It is clear from Fig.13 and Table 6 that the trained BiLSTM-based CSIE using Adadelta optimisation algorithm and SSE loss function achieves the best SER performance and provides the highest accuracy with $100 \%$. On the other hand, the same estimator achieves the lowest SER performance and provides accuracy with $97.46 \%$ using SGdm optimization algorithm and SSE loss function. This, in turn, shows the importance of studying the training process efficiency using different optimization algorithms in the case of using a specific loss function.

\section{CONCLUSIONS and FUTURE WORK}

The proposed DL-BiLSTM-based CSIE is an online pilot-assisted estimator. It is robust against a limited number of pilots and exhibits superior performance compared with conventional estimators; it is also robust under the conditions of a priori uncertainty of communication channel statistics (non-Gaussian/stationary statistical channels) and demonstrates superior performance compared with conventional estimators and DL LSTM NN-based CSIEs.

Two customized classification layers using the loss functions (MAE and SSE) are introduced. The proposed CSIE exhibits a consistent performance at large and small pilot numbers and superior performance at low SNRs, especially at limited pilots, compared with conventional estimators. It

PeerJ Comput. Sci. reviewing PDF | (CS-2021:04:60719:2:1:NEW 17 Jul 2021) 
440 also achieves the highest accuracy amongst all examined estimators at 64, 8, and 4 pilots for all 441 the used loss functions.

442 The proposed BiLSTM- and LSTM-based estimators have high prediction accuracies of 98.61\% 443 to $100 \%$ and $97.88 \%$ to $99.99 \%$, respectively, when using crossentropyex, MAE, and SSE loss 444 functions for 64,8 , and 4 pilots. The proposed BiLSTM using (Adam, and crossentroyex), 445 BiLSTM using (Adam, MAE, and SSE; and Adadelta, and SSE), and BiLSTM using (Adam, and 446 SSE), achieve the best SER performance and provide accuracies with $100 \%$ at 64 , 8, and 4 pilots 447 respectively. The proposed estimator is promising for $5 \mathrm{G}$ and beyond wireless communication 448 systems.

449 For future work, authors suggest the following research plans:

450 1. Investigating the proposed estimator's performance and accuracy by using different cyclic prefix $451 \quad$ lengths and types.

452 2. Developing robust loss functions by using robust statistics estimators, such as Tukey, Cauchy, 453 Huber and Welsh.

454 3. Investigating the performance of $\mathrm{CNN}$-, gated recurrent unit (GRU)- and simple recurrent unit

455

456

457

458

459

460

461

462

463

464

465

466

467

468

469

470

471

472

473

474

475

476

477

478

479

480

481

482

483

484

485

486

(SRU)-based CSIEs whilst using crossentropyex, MAE and SSE loss functions and for 64, 8 and 4 pilots.

\section{Acknowledgements}

The authors would like to acknowledge the financial support received from Taif University Researchers Supporting Project Number (TURSP-2020/61), Taif University, Taif, Saudi Arabia.

\section{References}

2019. 3GPP. TR38.901 Study on Channel Model for Frequencies from 0.5 to $100 \mathrm{GHz}$; $3 \mathrm{GPP}$ : Sophia Antipolis, France, 2019.

Bogdanovich V, Vostretsov AJJoCT, and Electronics. 2009. Application of the invariance and robustness principles in the development of demodulation algorithms for wideband communications systems. 54:1283-1291.

Dogo E, Afolabi O, Nwulu N, Twala B, and Aigbavboa C. 2018. A comparative analysis of gradient descent-based optimization algorithms on convolutional neural networks. 2018 International Conference on Computational Techniques, Electronics and Mechanical Systems (CTEMS): IEEE. p 92-99.

Essai Ali MH. Deep learning-based pilot-assisted channel state estimator for OFDM systems. IET Communications 15:257-264. https://doi.org/10.1049/cmu2.12051

Hochreiter S, and Schmidhuber J. 1997. Long Short-Term Memory. 9:1735-1780. 10.1162/neco.1997.9.8.1735

Janocha K, and Czarnecki WMJapa. 2017. On loss functions for deep neural networks in classification.

Jeya R, Amutha B, Nikhilesh N, and Immaculate RRJs. 2019. Signal Interferences in Wireless Communication-An Overview. 2:3.

Joo J, Park MC, Han DS, and Pejovic VJIA. 2019. Deep learning-based channel prediction in realistic vehicular communications. IEEE Access 7:27846-27858.

Kang J-M, Chun C-J, and Kim I-MJIA. 2020. Deep Learning Based Channel Estimation for MIMO Systems With Received SNR Feedback. IEEE Access 8:121162-121181.

Karra K, Kuzdeba S, and Petersen J. 2017. Modulation recognition using hierarchical deep neural networks. 2017 IEEE International Symposium on Dynamic Spectrum Access Networks (DySPAN). p 1-3.

PeerJ Comput. Sci. reviewing PDF | (CS-2021:04:60719:2:1:NEW 17 Jul 2021) 
487 Kim H. 2015. Wireless communications systems design: John Wiley \& Sons.

488 Le Ha A, Van Chien T, Nguyen TH, and Choi W. 2021. Deep Learning-Aided 5G Channel

489

490

491

492

493

494

495

496

497

498

499

500

501

502

503

504

505

506

507

508

509

510

511

512

513

514

515

516

517

518

519

520

521

522

523

524

525

526

527

528

529

530

531

532 Estimation. 2021 15th International Conference on Ubiquitous Information Management and Communication (IMCOM): IEEE. p 1-7.

Liao Y, Hua Y, Dai X, Yao H, and Yang X. 2019. ChanEstNet: A deep learning based channel estimation for high-speed scenarios. ICC 2019-2019 IEEE International Conference on Communications (ICC): IEEE. p 1-6.

Luo C, Ji J, Wang Q, Chen X, Li PJIToNS, and Engineering. 2018a. Channel state information prediction for $5 \mathrm{G}$ wireless communications: A deep learning approach. 7:227-236.

Luo C, Ji J, Wang Q, Chen X, Li PJIToNS, and Engineering. 2018b. Channel state information prediction for 5G wireless communications: A deep learning approach.

Ma X, Ye H, and Li Y. 2018. Learning Assisted Estimation for Time- Varying Channels. 2018 15th International Symposium on Wireless Communication Systems (ISWCS). p 1-5.

O'Shea T, Karra K, and Clancy TC. 2017. Learning approximate neural estimators for wireless channel state information. 2017 IEEE 27th international workshop on machine learning for signal processing (MLSP): IEEE. $\mathrm{p}$ 1-7.

Ong T. 2017. Facebook's translations are now powered completely by AI.

Oyerinde OO, and Mneney SH. 2012a. Review of Channel Estimation for Wireless Communication Systems. IETE Technical Review 29:282-298. 10.4103/02564602.101308

Oyerinde OO, and Mneney SHJITr. 2012b. Review of channel estimation for wireless communication systems. IETE Technical Review 29:282-298.

Ponnaluru S, and Penke S. 2020. Deep learning for estimating the channel in orthogonal frequency division multiplexing systems. Journal of Ambient Intelligence and Humanized Computing. 10.1007/s12652-020-02010-1

Sarwar A, Shah SM, and Zafar I. 2020. Channel Estimation in Space Time Block Coded MIMOOFDM System using Genetically Evolved Artificial Neural Network. 2020 17th International Bhurban Conference on Applied Sciences and Technology (IBCAST): IEEE. p 703-709.

Senol H, Tahir ARB, and Özmen AJTS. 2021. Artificial neural network based estimation of sparse multipath channels in OFDM systems. Telecommunication Systems:1-10.

Sharma R, Vinutha M, and Moharir M. 2016. Revolutionizing machine learning algorithms using gpus. 2016 International Conference on Computation System and Information Technology for Sustainable Solutions (CSITSS): IEEE. p 318-323.

Sheikh AUH. 2004. Interference, Distortion and Noise. Wireless Communications: Theory and Techniques. Boston, MA: Springer US, 225-285.

Soydaner DJIJoPR, and Intelligence A. 2020. A comparison of optimization algorithms for deep learning. 34:2052013.

Wu Y, Schuster M, Chen Z, Le QV, Norouzi M, Macherey W, Krikun M, Cao Y, Gao Q, and Macherey KJapa. 2016. Google's neural machine translation system: Bridging the gap between human and machine translation.

Yang Y, Gao F, Ma X, and Zhang S. 2019a. Deep Learning-Based Channel Estimation for Doubly Selective Fading Channels. IEEE Access 7:36579-36589. 10.1109/ACCESS.2019.2901066

Yang Y, Gao F, Ma X, and Zhang SJIA. 2019b. Deep learning-based channel estimation for doubly selective fading channels. 7:36579-36589.

Peer) Comput. Sci. reviewing PDF | (CS-2021:04:60719:2:1:NEW 17 Jul 2021) 
533 Ye H, Li GY, and Juang B. 2018. Power of Deep Learning for Channel Estimation and Signal 534 Detection in OFDM Systems. IEEE Wireless Communications Letters 7:114-117. $535 \quad$ 10.1109/LWC.2017.2757490

536 Zeiler MDJapa. 2012. Adadelta: an adaptive learning rate method.

537 Zhao C, Huang X, Li Y, and Yousaf Iqbal MJS. 2020. A Double-Channel Hybrid Deep Neural $538 \quad$ Network Based on CNN and BiLSTM for Remaining Useful Life Prediction. 20:7109.

539 Zhou R, Liu F, and Gravelle CW. 2020. Deep Learning for Modulation Recognition: A Survey 540 541 With a Demonstration. IEEE Access 8:67366-67376. 10.1109/ACCESS.2020.2986330 
Figure 1

Long short-term memory (LSTM) cell.

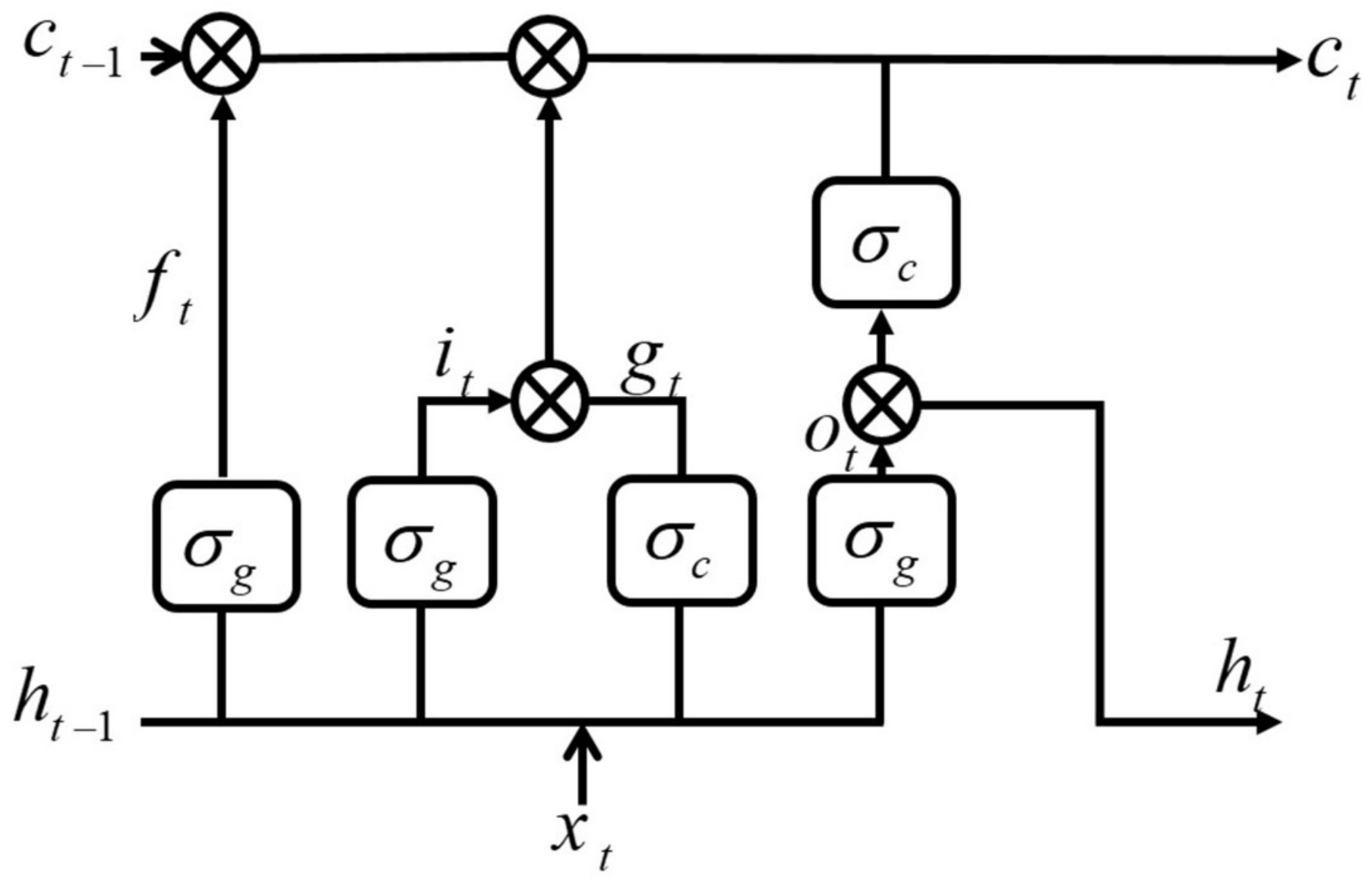


Figure 2

BiLSTM-NN architecture.

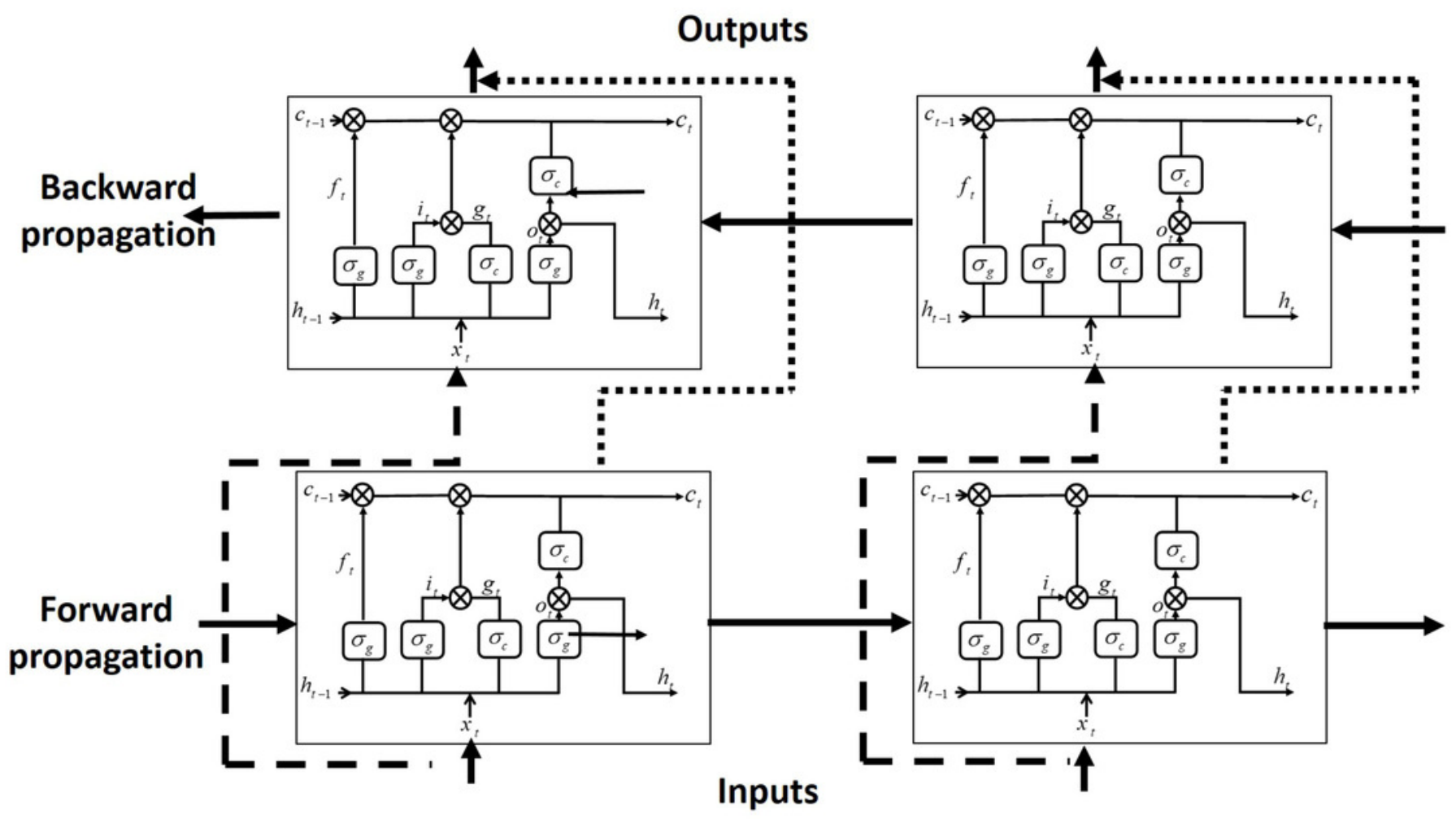


Figure 3

Structure of the DL BiLSTM NN for the BiLSTM estimator. 
Sequence input layer with 256

dimensions

(256 activation functions)

BiLSTM Layer with $30 \mathrm{H}$. N.

(60 activation functions)

Input weights (240x256)

Recurrent weights (240x30)

Bias (240x1)

Full connected layer with 4 dimensions

(4 activation functions)

Weights $(4 \times 60)$

Bias $(4 \times 1)$

\section{Softmax layer \\ (4 activation functions)}

\section{Classification output layer with} (crossentropy or MAE or SSE) loss

\section{function}


Figure 4

Offline training of the BiLSTM-NN-based CSI estimator. 


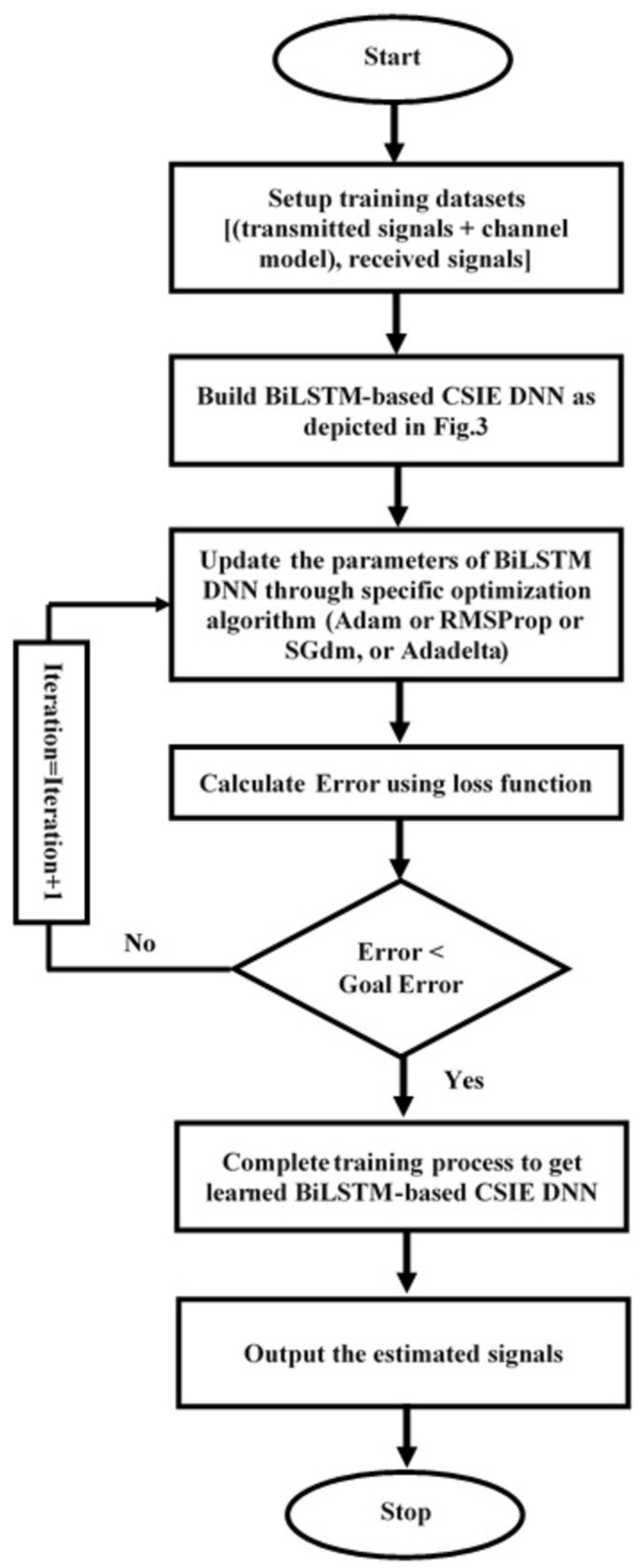


Figure 5

Conventional OFDM system
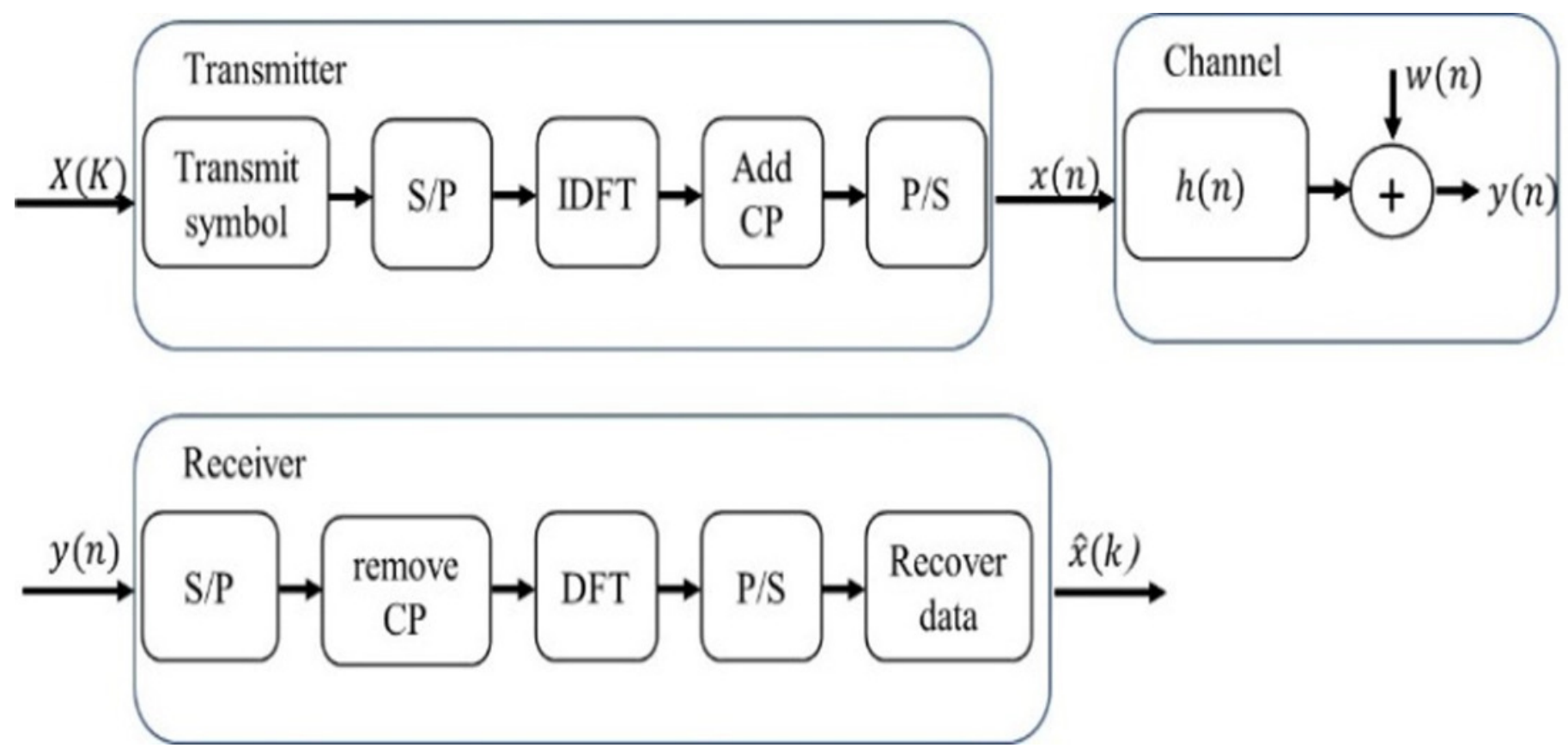


\section{Figure 6}

SER comparison of LS, MMSE, BiLSTM and LSTM estimators using 64 pilots, the Adam learning algorithm and crossentropyex, MAE and SSE loss functions.

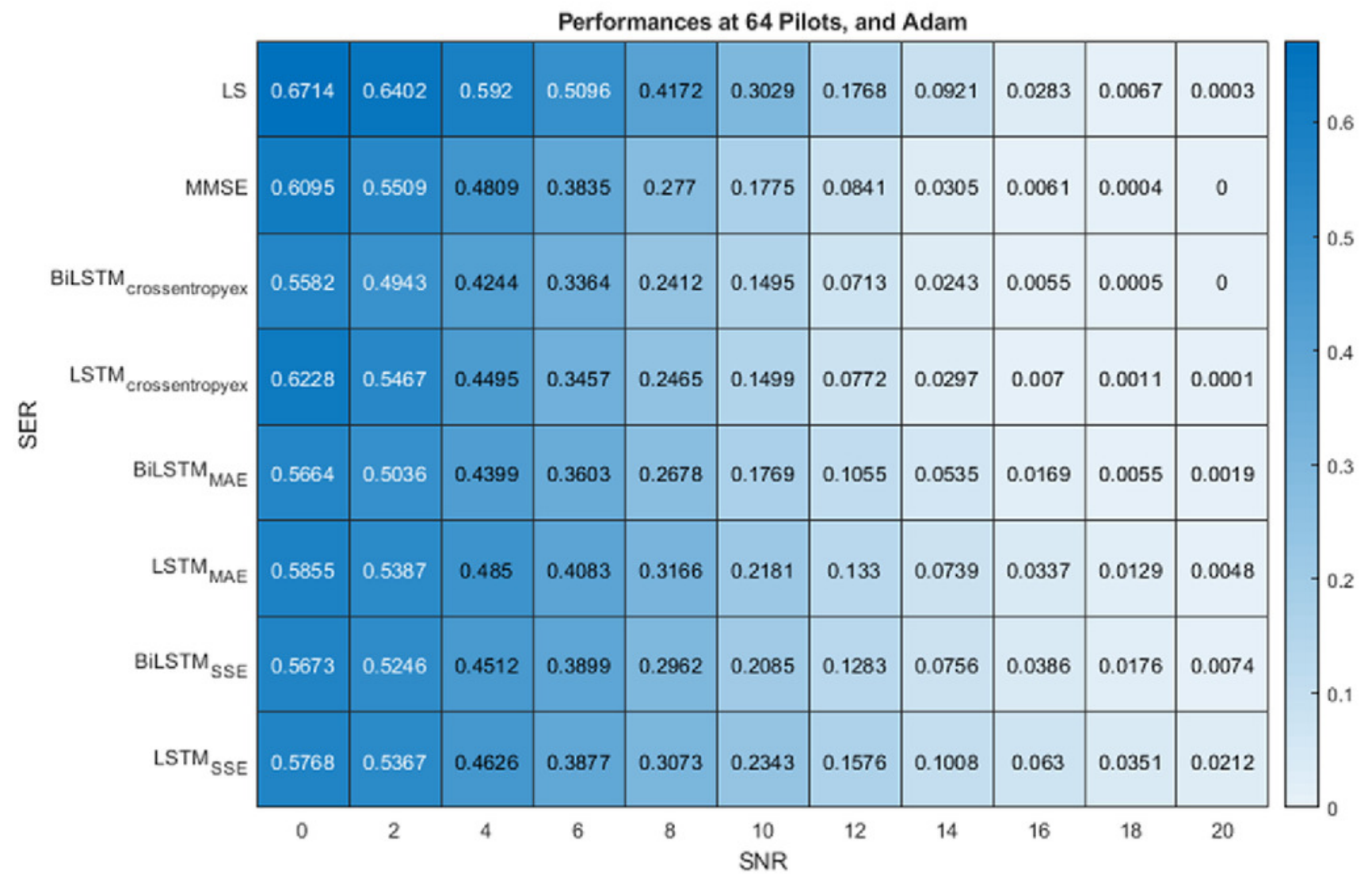




\section{Figure 7}

SER performance comparison of LS, MMSE, BiLSTM, and LSTM estimators using 8 pilots, the Adam learning algorithm and crossentropyex, MAE and SSE loss functions.

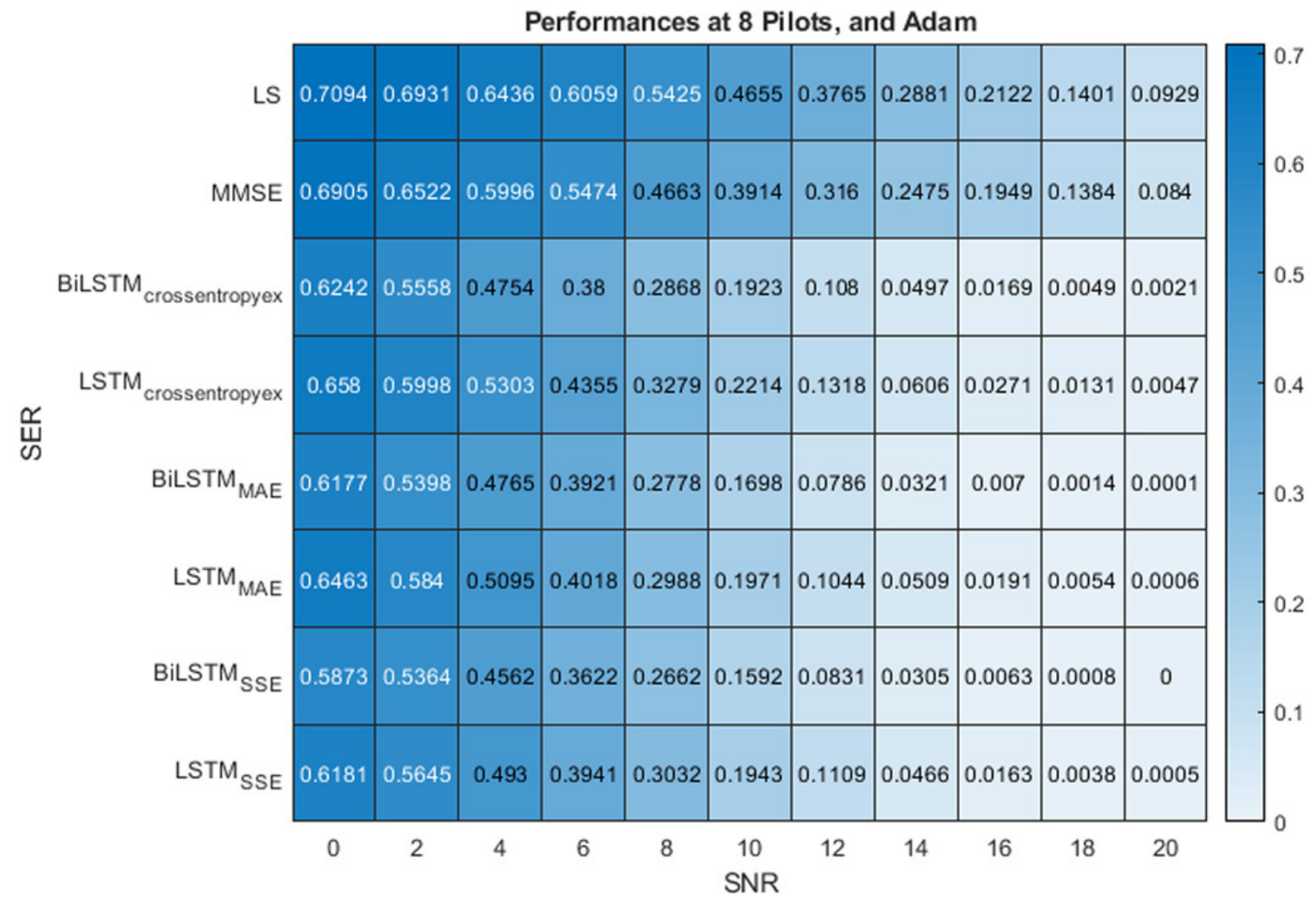




\section{Figure 8}

SER performance comparison of LS, MMSE, BiLSTM, and LSTM estimators using 4 pilots, the Adam learning algorithm and crossentropyex, MAE and SSE loss functions.

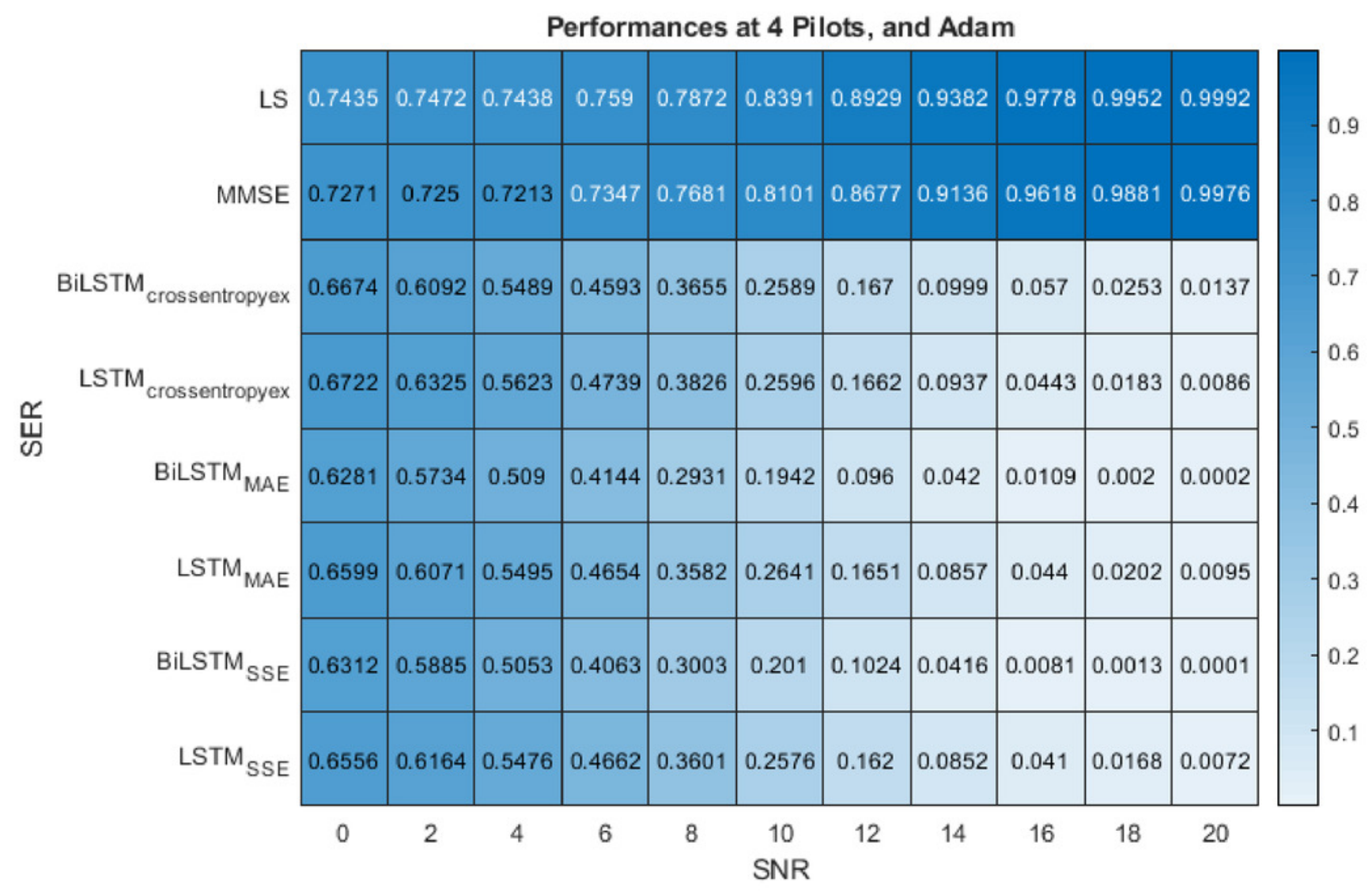


Figure 9

SER performance comparison of the best DL BiLSTM-based CSIEs using various pilots and loss functions.

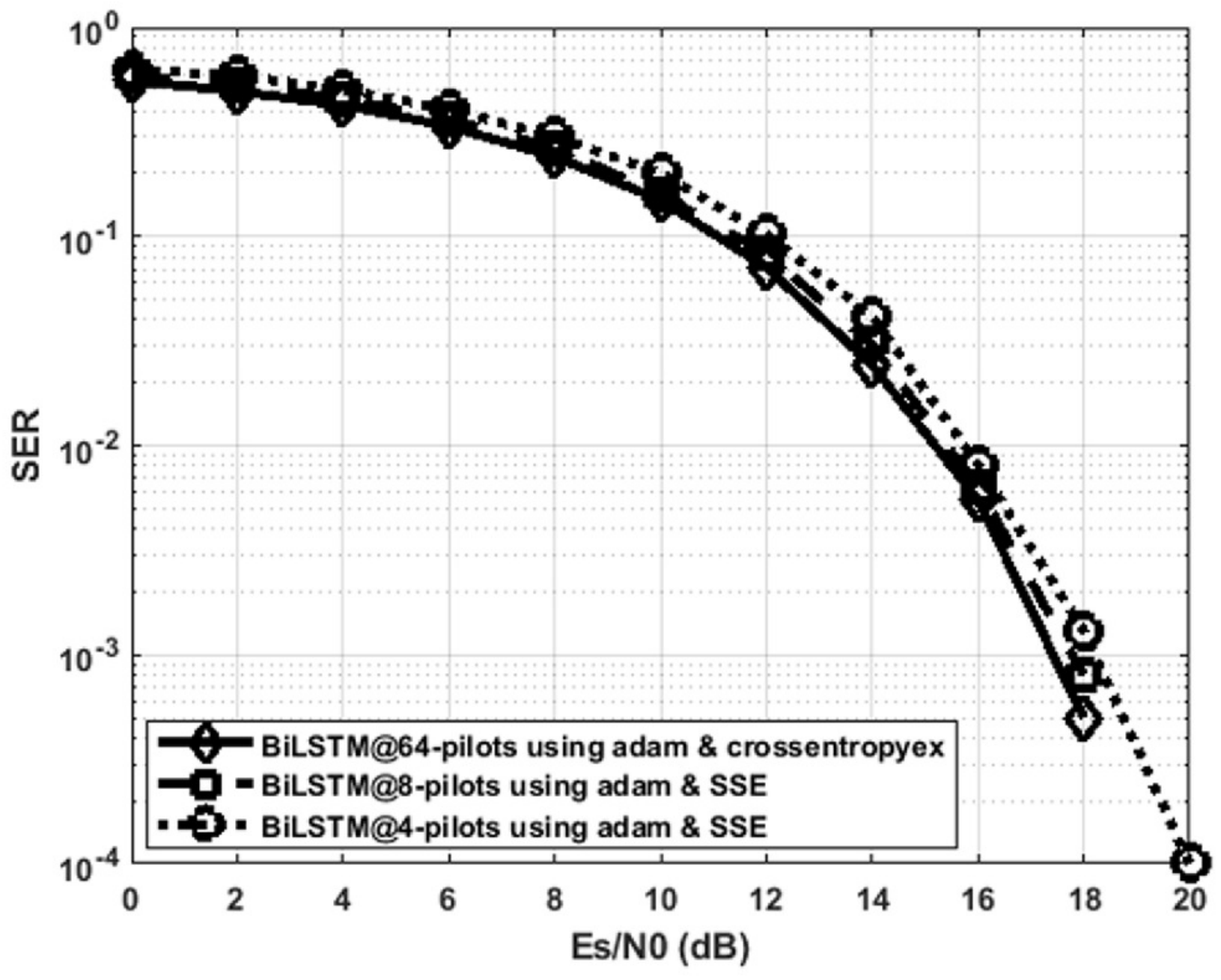


Figure 10

Loss curves comparison of BiLSTM- and LSTM- based estimators using 64 pilots, the Adam learning algorithm and crossentropyex, MAE and SSE loss functions.

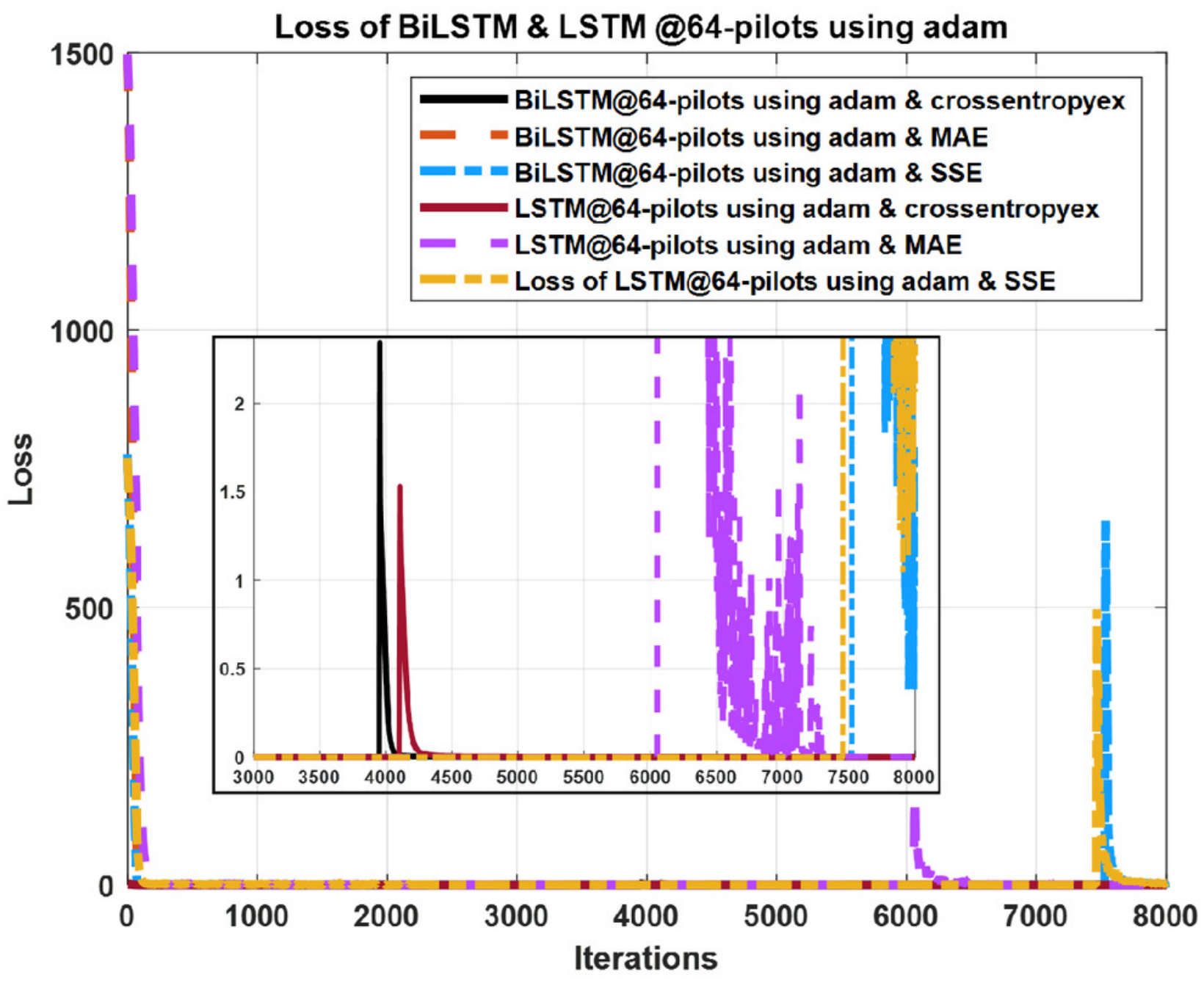


Figure 11

Loss curves comparison of BiLSTM- and LSTM-based estimators using 8 pilots, the Adam learning algorithm and crossentropyex, MAE and SSE loss functions.

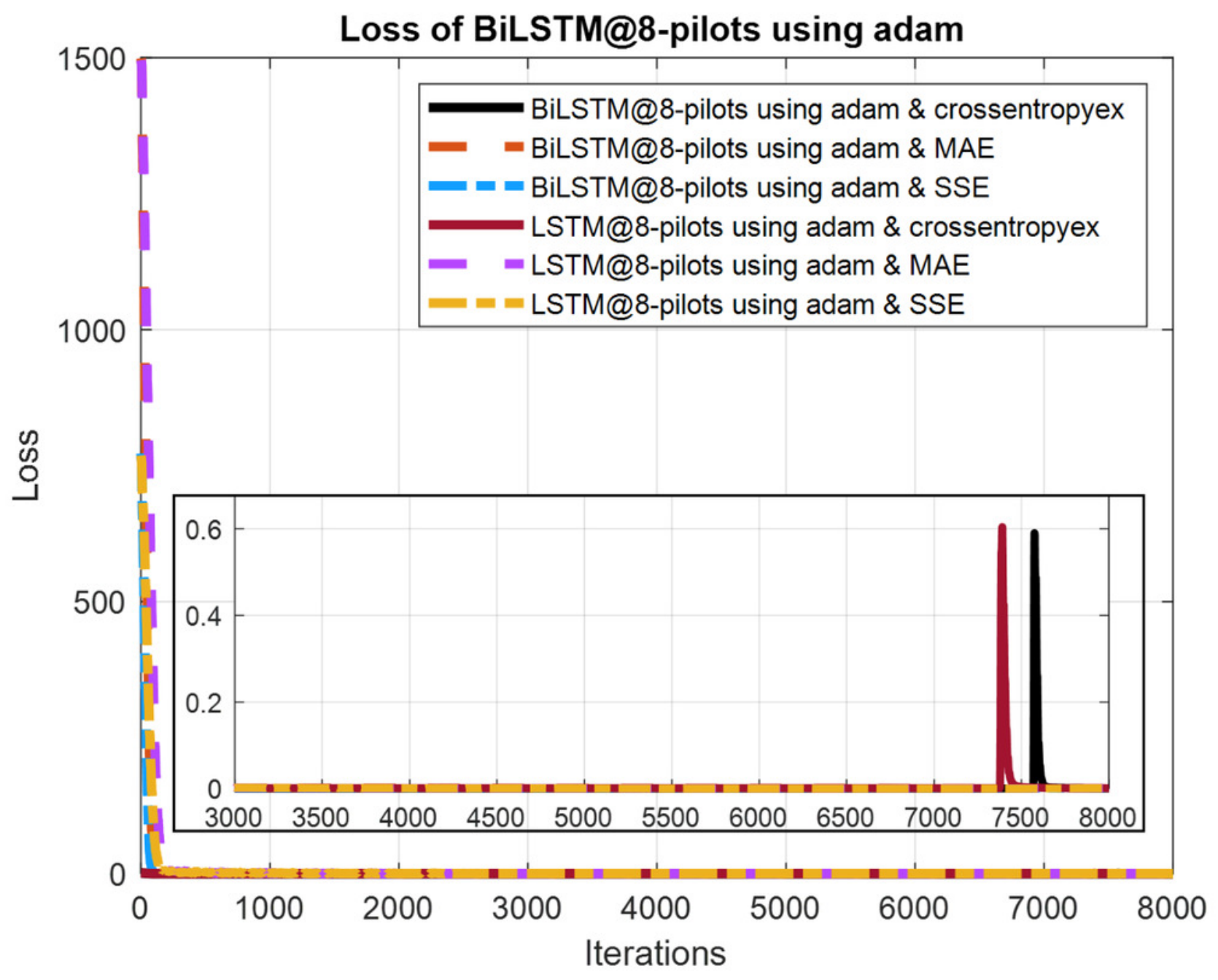


Figure 12

Loss curves comparison of BiLSTM- and LSTM-based estimators using 4 pilots, the Adam learning algorithm and crossentropyex, MAE and SSE loss functions.

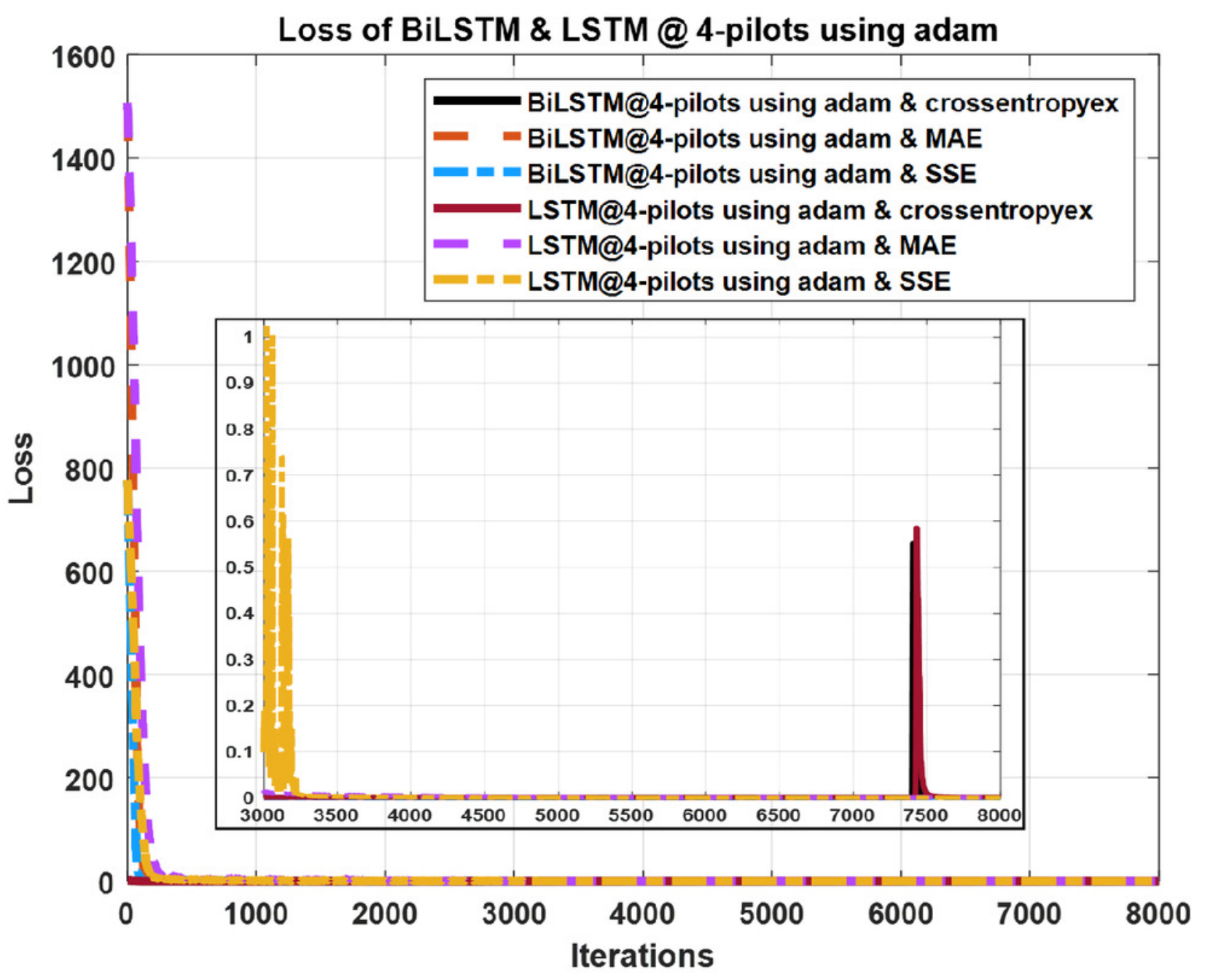




\section{Figure 13}

Performance comparison of BiLSTM-based estimator using 8 pilots, the RMSProp, SGdm, and Adadelta optimisation algorithms and crossentropyex, MAE and SSE loss functions.

\begin{tabular}{|c|c|c|c|c|c|c|c|c|c|c|c|c|}
\hline \multirow[b]{2}{*}{ RMSProp $_{\text {crossentropyex }}$} & \multicolumn{11}{|c|}{ Performances of BiLSTM using 8 Pilots } & \\
\hline & 0.6333 & 0.564 & 0.4995 & 0.3858 & 0.2833 & 0.1881 & 0.1086 & 0.0436 & 0.0153 & 0.0045 & 0.001 & 0.6 \\
\hline RMSProp $_{\text {MAE }}$ & 0.6352 & 0.5739 & 0.5004 & 0.4068 & 0.3006 & 0.1967 & 0.1153 & 0.0533 & 0.0191 & 0.0055 & 0.0016 & \\
\hline RMSProp $_{S S E}$ & 0.6183 & 0.5564 & 0.4781 & 0.3896 & 0.3013 & 0.1979 & 0.1161 & 0.0637 & 0.0269 & 0.0098 & 0.0026 & \\
\hline SGdm $_{\text {crossentropyex }}$ & 0.6355 & 0.5693 & 0.5016 & 0.4208 & 0.3308 & 0.2382 & 0.1518 & 0.0896 & 0.0507 & 0.0262 & 0.0147 & 0.4 \\
\hline $\mathrm{SGdm}_{\mathrm{MAE}}$ & 0.6334 & 0.5756 & 0.5066 & 0.4169 & 0.3278 & 0.2312 & 0.1559 & 0.0893 & 0.048 & 0.0255 & 0.0124 & \\
\hline $\mathrm{SGdm}_{\mathrm{SSE}}$ & 0.6465 & 0.5814 & 0.5108 & 0.4327 & 0.342 & 0.2478 & 0.1678 & 0.1031 & 0.0605 & 0.0406 & 0.0254 & \\
\hline Adadelta crossentropyex & 0.6216 & 0.5577 & 0.4694 & 0.3647 & 0.2728 & 0.1743 & 0.0924 & 0.0316 & 0.0094 & 0.0019 & 0.0002 & .2 \\
\hline Adadelta ${ }_{M A E}$ & 0.6256 & 0.57 & 0.4926 & 0.3961 & 0.2884 & 0.1884 & 0.0944 & 0.033 & 0.0107 & 0.0008 & 0 & 0.1 \\
\hline Adadelta $_{\mathrm{SSE}}$ & 0.5815 & 0.5154 & 0.4497 & 0.3525 & 0.2505 & 0.1624 & 0.0798 & 0.0293 & 0.0051 & 0.0002 & 0.0001 & \\
\hline & 0 & 2 & 4 & 6 & 8 & $\begin{array}{c}10 \\
\text { SNR }\end{array}$ & 12 & 14 & 16 & 18 & 20 & \\
\hline
\end{tabular}




\section{Table $\mathbf{1}$ (on next page)}

BiLSTM- and LSTM-NN structure parameters and training process options 


\begin{tabular}{cc}
\hline Parameter & Value \\
\hline Input Size & 256 \\
\hline BiLSTM Layer Size & 30 hidden neurons \\
LSTM Layer Size & 30 hidden neurons \\
\hline
\end{tabular}

\begin{tabular}{cc}
\hline FC Layer Size & 4 \\
\hline Loss Functions & Crossentropyex, MAE, SSE \\
\hline
\end{tabular}

\begin{tabular}{cc}
\hline Mini Batch Size & 1000 \\
\hline Epochs Number & 1000 \\
\hline Learning Algorithm & Adam \\
\hline Training Data Size & 8000 - OFDM frame \\
\hline Validation Data Size & 2000 - OFDM frame \\
\hline Test Data Size & 10000 - OFDM frame \\
\hline
\end{tabular}




\section{Table 2 (on next page)}

OFDM system and channel parameters 
35

36

\begin{tabular}{cc}
\hline Parameter & Value \\
\hline $\begin{array}{c}\text { Modulation } \\
\text { Mode }\end{array}$ & QPSK \\
\hline $\begin{array}{c}\text { Carrier } \\
\text { Frequency }\end{array}$ & $2.6 \mathrm{GHz}$ \\
\hline Paths Number & 24 \\
\hline CP Length & 16 \\
\hline $\begin{array}{c}\text { Subcarrier } \\
\text { Number }\end{array}$ & 64 \\
\hline Pilot Number & 64,8 and 4 \\
\hline
\end{tabular}




\section{Table $\mathbf{3}$ (on next page)}

Accuracy comparison of the examined estimators using 64 pilots 
12

13

14

15

16

\begin{tabular}{ccccc}
\hline \multicolumn{5}{c}{ 64 pilots } \\
\hline & BiLSTM & LSTM & MMSE & LS \\
\hline Crossentropyex & 100 & 99.99 & 100 & 99.94 \\
\hline SSE & 99.23 & 97.88 & 100 & 99.96 \\
\hline MAE & 99.87 & 99.52 & 100 & 99.97 \\
\hline
\end{tabular}

17

18

19

20

21

22 


\section{Table 4 (on next page)}

Accuracy comparison of the examined estimators using 8 pilots 
10

\begin{tabular}{ccccc}
\hline \multicolumn{5}{c}{ 8 pilots } \\
\hline & BiLSTM & LSTM & MMSE & LS \\
\hline Crossentropyex & 99.84 & 99.53 & 91.34 & 91.62 \\
\hline SSE & 100 & 99.95 & 91.60 & 91.49 \\
\hline MAE & 100 & 99.94 & 91.53 & 91.50 \\
\hline
\end{tabular}

11

12

13

14

15

16

17 


\section{Table 5 (on next page)}

Accuracy comparison of the examined estimators using 4 pilots 


\section{Table 6(on next page)}

Performance comparison of different optimisation algorithms and its related accuracies 


\begin{tabular}{ccc}
\hline Order & Optimisation algorithm $_{\text {Loss function }}$ & Accuracy \\
\hline First & Adadelta $_{\text {SSE }}$ & $100 \%$ \\
\hline Second & Adadelta $_{\text {crossentropyex }}$ & $99.99 \%$ \\
\hline Third & Adadelta $_{\text {MAE }}$ & $99.98 \%$ \\
\hline Fourth & RMSProp $_{\text {crossentropyex }}$ & $99.90 \%$ \\
\hline Fifth & RMSProp $_{\text {MAE }}$ & $99.84 \%$ \\
\hline Sixth & RMSProp $_{\text {SSE }}$ & $99.74 \%$ \\
\hline Seventh & SGdm $_{\text {MAE }}$ & $98.76 \%$ \\
\hline Eighth & SGdm $_{\text {crossentropyex }}$ & $98.53 \%$ \\
\hline Ninth & SGdm $_{\text {SSE }}$ & $97.46 \%$ \\
\hline
\end{tabular}

11

12

13

14

15 